\title{
Integrierte Lagersystemplanung
}

\author{
Thomas Atz, M.Sc., Prof. Dr.-Ing. W. A. Günthner \\ Technische Universität München \\ Lehrstuhl für Fördertechnik Materialfluss Logistik fml
}

\begin{abstract}
Der Begriff "Integrierte Lagersystemplanung" steht für eine ganzheitliche Planungsmethode für automatische Lagersysteme, die es erlaubt, unterschiedliche technische Lagerkonfigurationen in Kombination mit verwendeten Lagerstrategien zu untersuchen und zu vergleichen. Dazu wird die Berechnung der Umschlagsleistung und der Kosten in einem Rechenwerkzeug zusammengeführt und mittels einer mathematischen Optimierung nach dem optimal dimensionierten Lager für einen spezifischen Anwendungsfall gesucht.
\end{abstract}

\section{$1 \quad$ Einleitung}

Die Auslegung automatischer Lagersysteme mit schienengeführten Regalbediengeräten hängt von einer Vielzahl an Parametern ab, welche sich aus Lagerkonfiguration und Lagerstrategie ergeben. Die wichtigsten Kenngrößen bei der Auslegung eines Lagersystems sind Stellplatzanzahl, Umschlagsleistung und Investitionsbedarf. Stellplatzanzahl und Umschlagsleistung sind Planungsvorgaben, welche sich aus unternehmensspezifischen Faktoren ableiten. Die zu realisierende Stellplatzanzahl ist von der Artikelstruktur, den Produktionslosgrößen und den Sicherheitsbeständen abhängig. Die Umschlagsleistung hängt von den Materialflüssen pro Zeiteinheit, die von den vor- und nachgelagerten Unternehmensbereichen in oder aus dem Lager führen, ab. Zielgröße einer Planung ist der Investitionsbedarf, welcher aus einer ausgearbeiteten Planungsvariante abgeleitet wird und i. d. R. möglichst gering sein soll.

Die herkömmliche Planungsvorgehensweise ist sequenziell und bedingt eine getrennte Betrachtung der drei Kenngrößen. In der Planung wird zunächst ein Groblayout erarbeitet, welches eine ausreichende Anzahl an Stellplätzen vorsieht und in einem zweiten Schritt bezüglich der Umschlagsleistung bewertet wird. Oftmals fehlen dazu jedoch geeignete Berechnungsmodelle. Beispielsweise ist der Effekt erhöhter Umschlagsleistungen durch intelligente Lagerorganisationen zwar bekannt, aber gleichzeitig sehr schwer für die Vielzahl an technischen Lagerkonfigurationen quantifizierbar. Nach der Bestimmung der Stellplatzkapazität und der Umschlagsleistung werden der Investitionsaufwand abgeschätzt und, bei schlechter Wirtschaftlichkeit, in iterativen Schritten Änderungen am Layout oder der technischen Ausführung vollzogen. Diese Vorgehensweise erfordert Expertise und Erfahrung vom Planer und verursacht einen hohen Aufwand, um das Leistungs- und Kostenoptimum einer Planungsvariante zu finden. In der Grobplanungsphase können aus Zeit- und Kostengründen nur einige wenige technische Varianten untersucht werden. Die durch die Untersuchung ermittelte geeignetste Variante wird in der 
Feinplanungsphase detailliert ausgearbeitet und oftmals simulativ untersucht. Eine Simulation erlaubt es, Einflüsse zu berücksichtigen, die eigentlich schon zur Auswahl in der Grobplanungsphase wertvoll gewesen wären. Das Ergebnis der schwierigen und aufwendigen Planung sind suboptimale automatische Lagersysteme, welche nicht immer für den jeweiligen Anwendungsfall richtig dimensioniert sind.

Das Forschungsprojekt „Integrierte Lagersystemplanung“ verfolgt das Ziel, den Planer in der Grobplanungsphase zu unterstützen, eine neue ganzheitliche Planungsvorgehensweise zu entwickeln und in einem Rechenwerkzeug umzusetzen. Dieses erlaubt es, mit geringem Zeitaufwand eine große Bandbreite an teilweise auch komplexen Planungsvarianten zu betrachten.

\section{Automatische Lagersysteme}

Automatisierte Hochregallager üben eine Lager-, Speicher- oder Puffertätigkeit aus und sind i.d.R. über eine Lagervorzone an die Unternehmensbereiche Produktion, Kommissionierung, Warenein- und Warenausgang angebunden. Sie können im Allgemeinen in drei Kategorien untergliedert werden: Automatische Palettenhochregallager (HRL) werden für Lagergut, welches sich auf EURO- oder Industriepaletten befindet, verwendet. Das Gewicht dieser Paletten liegt meist zwischen 300 und $3000 \mathrm{~kg}$. Palettenhochregallager können bis zu einer Höhe von $50 \mathrm{~m}$ gebaut werden. Während bei einer niedrigen Höhe meist die Hallenbauweise zum Einsatz kommt, hat sich bei größeren Höhen ab ca. $18 \mathrm{~m}$ die Silobauweise mit tragenden Regalen durchgesetzt. Bei Tablarlagern werden Tablare als Ladehilfsmittel verwendet, auf welche mehrere Lagergüter angeordnet werden. Es können meist Gewichte von bis zu $300 \mathrm{~kg}$ pro Tablar erreicht werden. Automatische Kleinteilelager (AKL) werden für bis zu $50 \mathrm{~kg}$ leichte und relativ kleine Ladeeinheiten (LE) verwendet. Diese befinden sich meist in genormten Behältern oder Kartons.

In den vergangenen Jahrzehnten sind eine Vielzahl an Lagerkonfigurationen entstanden um den unterschiedlichen Anforderungen der modernen Logistik gerecht zu werden. Die Lagerkonfiguration beschreibt dabei den physischen Aufbau des Lagers. Die Ausprägungen gängiger Lagerkonfigurationen sind in Tabelle 1 dargestellt.

\begin{tabular}{|l|l|l|l|}
\hline Lagerkonfigurationsparameter & Ausprägungsformen & \multicolumn{2}{|l|}{ doppeltief } \\
\hline Lagertiefe & einfachtief & \multicolumn{2}{|l|}{3} \\
\hline Anzahl Lastaufnahmemittel pro RBG & 1 & \multicolumn{2}{|l|}{ doppelbreit } \\
\hline Lastaufnahmemittel- / Gassenbreite & einfachbreit & \multicolumn{2}{|l|}{ mehrgassig } \\
\hline Wirkungsbereich des RBG & eingassig & \multicolumn{3}{|l|}{4} \\
\hline Anzahl Übergabepunkte pro Gasse & 2 & $\begin{array}{l}\text { In x- oder y-Richtung } \\
\text { verschoben }\end{array}$ \\
\hline Lage der Übergabepunkte & $\begin{array}{l}\text { In einem Eckpunkt des } \\
\text { Regals }\end{array}$ & \\
\hline
\end{tabular}

Tabelle 1: Übersicht wichtiger Lagerkonfigurationsparameter und Ausprägungsmöglichkeiten

\footnotetext{
${ }^{1}$ Auch als Gassentiefe bezeichnet. 
Eine einfache und oft realisierte Lagerkonfiguration ist das einfachtiefe Lager, das von gassengebundenen Regalbediengeräten (RBG) mit jeweils einem einfachfachbreiten Lastaufnahmemittel (LAM) bedient wird. Je nach Anforderungen bezüglich Lagerkapazität und Durchsatzleistung können doppeltiefe Lagerformen mit mehr Stellplätzen bezogen auf das Lagervolumen, Mehrfachlastaufnahmemittel für eine höhere Umschlagsleistung oder kurvengängige Regalbediengeräte zur Bedienung von mehreren Gassen bei einer geringen benötigten Leistung, zum Einsatz kommen.

Jede Lagerkonfiguration kann mit mehreren Lagerstrategien betrieben werden, an denen sich der operative Ablauf der Arbeitsspiele orientiert. Diese Strategien bestimmen die Lagerplatzvergabe und das konkrete Verhalten eines RBG beim Zugriff auf Ladeeinheiten und sind i.d.R. auf die Lagerkonfiguration abgestimmt. Die Strategien werden an dieser Stelle nach inrem Wirkungsbereich gegliedert (Tabelle 2):

\begin{tabular}{|c|c|c|c|c|c|}
\hline Lagerstrategien & \multicolumn{5}{|c|}{ Ausprägungsformen } \\
\hline Einlagerstrategie & $\begin{array}{l}\text { zufällige } \\
\text { Einlagerung }\end{array}$ & $\begin{array}{l}\text { erung nahe } \\
\text { gerung }\end{array}$ & Zonierur & BC) & $\begin{array}{l}\text { Mehrfacheinlager } \\
\text { ung nahe } \\
\text { Auslagerung }\end{array}$ \\
\hline Umlagerstrategie & \multirow{2}{*}{\multicolumn{2}{|c|}{ keine Umlagerung }} & agerung & \multicolumn{2}{|c|}{$\begin{array}{l}\text { Umlagerung nahe } \\
\text { Auslagerung }\end{array}$} \\
\hline Auslagerstrategie & & & \multicolumn{3}{|c|}{ abgeschwächtes FIFO } \\
\hline Reihenfolgestrategie & \multicolumn{2}{|c|}{ keine Reihenfolgestrategie } & \multicolumn{3}{|c|}{ Fahrwegoptimierung } \\
\hline Ruhepositionsstrategie & \multicolumn{2}{|c|}{ Mit Rückkehr zum Aufnahme-Punkt } & \multicolumn{3}{|c|}{ Verweilen am letzten Aktionspunkt } \\
\hline $\begin{array}{l}\text { Bewegungsstrategie / } \\
\text { Spielart }\end{array}$ & Einlagerung & \multicolumn{2}{|c|}{ Auslagerung } & \multicolumn{2}{|c|}{ kombiniertes Spiel } \\
\hline
\end{tabular}

Tabelle 2: Übersicht wichtiger Lagerstrategien und Ausprägungsmöglichkeiten

Für die Praxis relevante Einlagerstrategien sind beispielsweise die zufällige Lagerplatzzuordnung $^{2}$, bei welcher eine einzulagernde Ladeeinheit in ein zufällig bestimmtes freies Lagerfach eingelagert wird, und die Einlagerung nahe Auslagerung, bei der das Einlagerfach möglichst nahe am nächsten Auslagerfach gewählt wird, um Fahrwege einzusparen.

Umlagerstrategien können im doppeltiefen Lager notwendig werden, wenn auf eine verdeckt stehende Ladeeinheit zugegriffen werden muss. In diesem Fall wird zuerst die vordere Ladeeinheit in ein anderes Lagerfach umgelagert.

Bei den Auslagerstrategien kommt in der Praxis meist ein strenges FIFO $^{3}$ zum Einsatz, um einer Überalterung des Lagerbestandes vorzubeugen. In einigen Branchen wird dieses Prinzip aufgeweicht und Artikel einer gemeinsamen Charge werden bei einer Auslagerung gleichberechtig behandelt. Für das RBG ergibt sich somit ein Einsparpotenzial mittels Fahrwegoptimierung durch geschickten Zugriff auf

\footnotetext{
${ }^{2}$ Auch als chaotische Lagerung bekannt.

${ }^{3}$ FIFO (First In First Out) bedeutet, dass der älteste Artikel zuerst ausgelagert wird. 
die Artikel. Weitere Reihenfolgestrategien können angewendet werden, wenn das RBG mehrere LAM besitzt.

\section{Umschlagsleistungsberechnung}

Die Umschlagsleistung beschreibt die Anzahl der Ein- und/oder Auslagerungen je Zeiteinheit. Sie ist in automatischen Lagersystemen abhängig von der Anzahl der RBG und von der Arbeitsspieldauer. Deren Berechnung ist seit über vier Jahrzehnten fester Bestandteil der Forschung auf dem Gebiet der Logistik. Eine präzise Schätzung der Leistungsfähigkeit eines Lagersystems ist das Fundament einer genauen Planung und verhindert Über- oder Unterdimensionierung, die zu hohen Investitions- oder Folgekosten führen können.

Die Bedeutung der Lagersystemplanung und die Komplexität der Bestimmung der durchschnittlichen Arbeitsspieldauer hat dazu geführt, dass der Verein Deutscher Ingenieure [VDI73] [VDI98] und die Fédération européenne de la manutention [FEM95] Richtlinien erstellt haben, die den Leistungsvergleich verschiedener Geräte und deren Abnahme erleichtern und vereinheitlichen. Diese Richtlinien sind jedoch nur bedingt als Planungshilfsmittel zu verwenden, da nur einfache Lagerkonfigurationen berechnet werden können und lagerorganisatorische Einflussgrößen auf die Umschlagsleistung außer Acht gelassen werden. Neben den Richtlinien beschäftigt sich eine große Bandbreite an internationalen Veröffentlichungen mit der Untersuchung des Einflusses eines bestimmten Parameters oder von Parameterkombinationen auf die mittlere Spielzeit. Einen Literaturüberblick über die Durchsatzberechnung in automatischen Hochregallagern geben Sarker und Babu [Sar95], Johnson und Brandeau [Joh96] sowie Roodbergen [Roo09]. Ein mathematisch exaktes Modell, welches alle Parameter die die Spielzeit beeinflussen vereinigt, wurde noch nicht entwickelt.

\subsection{Einheitlicher Ansatz zur Bestimmung der Umschlagsleistung unterschiedlich ausgeprägter Lagersysteme}

Die integrierte Planungsvorgehensweise basiert auf einem einheitlichen, leicht anwendbaren Modell zur Bestimmung der Umschlagleistung eines automatschen Lagersystems, welches praxisrelevante Lagerkonfigurationen abdeckt und entsprechende Lagerstrategien integriert.

Die einfachste Form, ein solches Modell zu verwirklichen, bietet die Ablaufsimulation. Die Simulation hat durch ihre Flexibilität das Potenzial, sämtliche Konfigurationen und Strategien abzudecken, erfordert im Gegenzug aber einen relativ hohen Rechenaufwand. Bei der hohen Anzahl an Funktionsauswertungen, die eine Optimierung erfordert, ist dieser Umstand problematisch. Dieser Nachteil kann durch den Einsatz von neuronalen Netzen eliminiert werden [Kra11]. Durch die Vielzahl an Einflussparametern, die bei der Planungsvorgehensweise berücksichtigt werden, ist der Trainingsaufwand für diese Netze jedoch nicht mehr durchführbar. 
Das entwickelte Gesamtmodell basiert deshalb auf ineinander aufbauenden mathematischen Modellen, da diese sehr effizient berechnet werden können und trotzdem ein großes Spektrum an Einflussgrößen berücksichtigen. Das Gesamtmodell zur Umschlagsleistungsberechnung führt bestehende Modelle zusammen und vereinheitlicht und erweitert diese um fehlende Aspekte. Dabei werden Modelle zur Berechnung von Spielzeitkomponenten, der Spielzeit einer Regalgasse und der Spielzeit mehrerer abhängiger Regalgassen verwendet. Abbildung 1 stellt neben der Gliederung des Gesamtmodells auch die wichtigsten Einflussgrößen auf die Untermodelle dar.

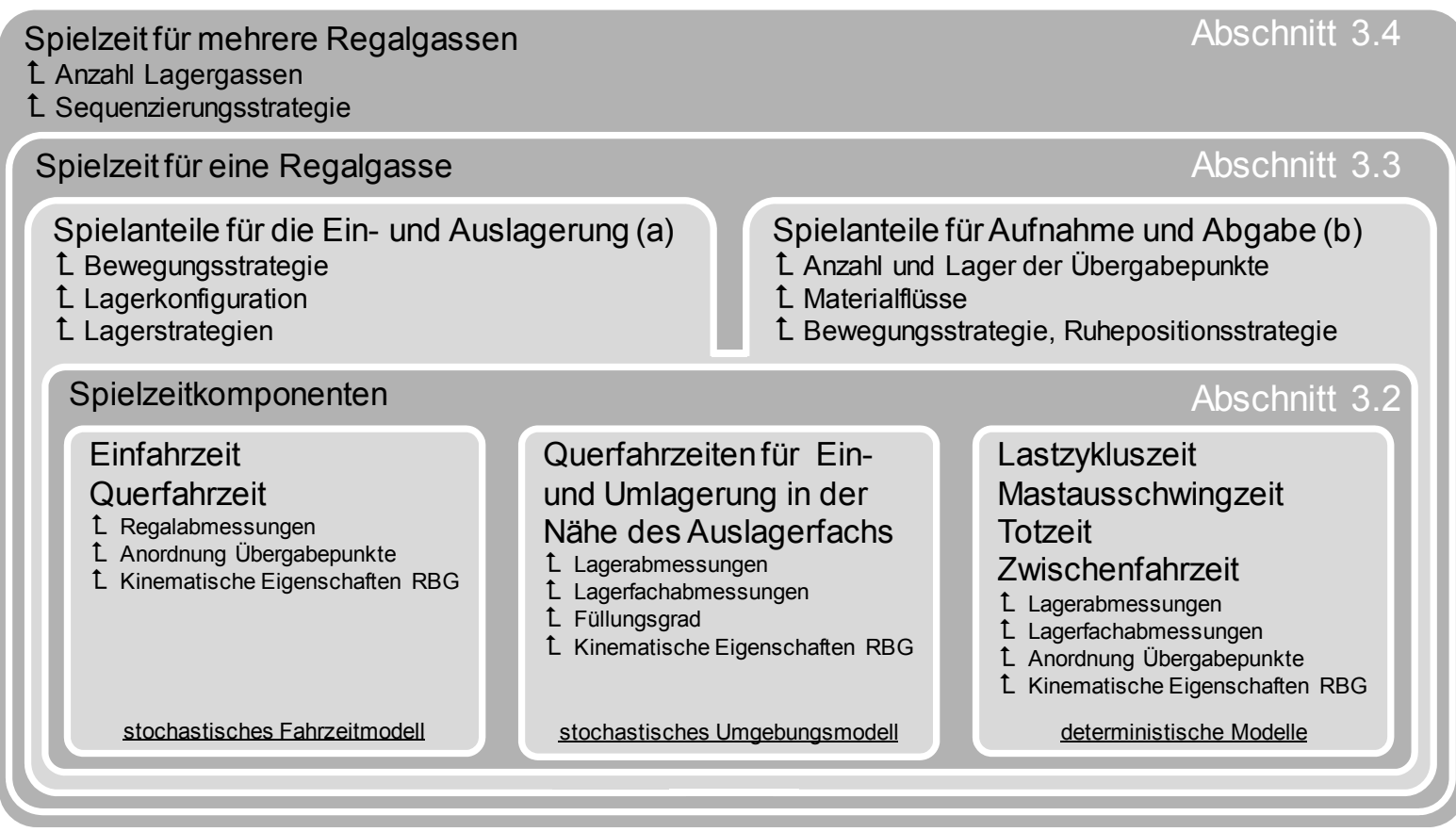

Abbildung 1: Berechnung der Umschlagsleistung eines automatischen Lagersystems; mathematisches Gesamtmodell mit Untermodellen und Einflussgrößen

Die Basis des Modells ist die Berechnung der Spielzeitkomponenten, welche typische Bewegungen des RBG abbilden. Die Einfahrzeit ist beispielsweise die mittlere Fahrzeit von einem Übergabepunkt am Rand der Regalwand zu einem beliebigen Lagerfach in der Regalwand. Die Querfahrzeit entspricht der mittleren Fahrzeit von einem beliebigen Lagerfach zu einem anderen beliebigen Lagerfach. Die Berechnung der Spielzeitkomponenten erfolgt mittels stochastischer und deterministischer Modelle und ist Ausgangsbasis zur Berechnung der mittleren Spielzeit eines RBG. Zur einfacheren Berechnung mehrerer aneinandergereihter Arbeitsspiele werden zwei Spielanteile unterschieden:

- Spielanteil (a) beschreibt im Wesentlichen die Vorgänge bei den Ein- und Auslagerungen. Dazu gehören Bewegungen des RBG und die eigentlichen Einund Aus- sowie eventuelle Umlagerungen. Der Spielanteil setzt sich aus Spielzeitkomponenten zusammen. Die Zusammensetzung ist dabei von der 
Lagerkonfiguration und Lagerstrategie sowie von der vom RBG gefahrenen Spielart (Einlagerung, Auslagerung oder kombiniertes Spiel) abhängig.

- Spielanteil (b) beschreibt die Vorgänge bei der Aufnahme und Abgabe der Lagereinheiten am Übergabepunkt. Die dafür notwendigen Bewegungen sind von der Spielart, der Ruhepositionsstrategie sowie der Lage der Übergabepunkte und den darüber führenden Materialflüssen innerhalb eines Zeitintervalls abhängig. Dieser Spielanteil umfasst Wechselfahrten zwischen den Übergabepunkten, die eigentliche Aufnahme und Abgabe sowie die Einfahrt ins Regal zum ersten (Einlager-) Fach oder die Ausfahrt aus dem Regal vom (Auslager-) Fach.

Die Spielzeitkomponenten sind aus bestehenden Berechnungsmethoden und Modellen abgeleitet, welche im folgenden Abschnitt 3.2 beschrieben werden. Die Zusammensetzung der Spielanteile und die Berechnung der Spielzeit werden im Abschnitt 3.3 und 3.4 erläutert.

\subsection{Berechnung einzelner Spielzeitkomponenten}

Die Spielzeitkomponenten können nach der ihrer Berechnung zugrundeliegenden Modellart untergliedert werden.

\section{Stochastisches Fahrzeitenmodell}

Zur Berechnung der Einfahr- und Querfahrzeit wird ein stochastisch-analytisches Modell nach der Idee von Bozer und White entwickelt [Boz84]. Dieses Modell beruht auf einem auf die Zeitkoordinaten normierten Regalwandmodell (Abbildung 2). Mittels eines stochastischen Ansatzes werden die mittleren Fahrzeiten für die Einfahrzeit (mittlere Fahrzeit vom Nullpunkt zu einem beliebigen Punkt in der Regalwand) und die Querfahrzeit (mittlere Fahrzeit von einem beliebigen Punkt zu einem anderen beliebigen Punkt) hergeleitet.

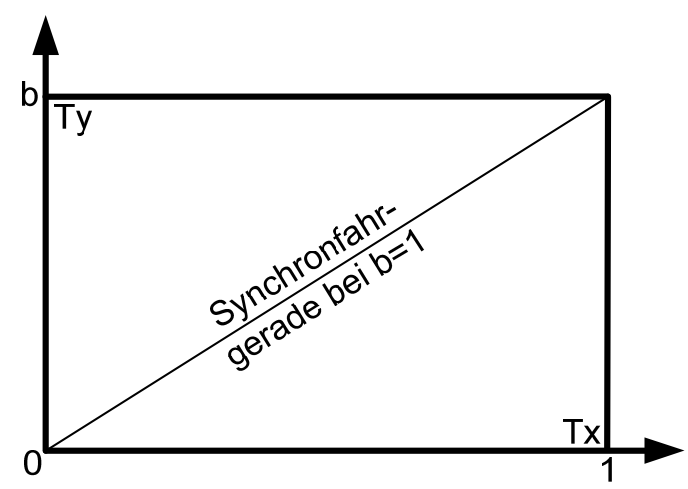

Abbildung 2: Fahrzeitmodell nach Bozer und White; Skizze einer normierten Regalwand

Die Anwendung des Modells besteht aus der Transformation der kartesischen Regalkoordinaten in Zeitkoordinaten und der Skalierung auf normierte Fahrzeiten. Anschließend werden die normierte mittlere Einfahrzeit und Querfahrzeit berechnet und in einem letzten Schritt denormiert. 
Dieses Fahrzeitenmodell hat gegenüber dem Modell der repräsentativen Punkte aus [VDI73] und [FEM95] den Vorteil, unabhängig vom Regalwandparameter b zu sein. Dieser Parameter spiegelt das Verhältnis zwischen Regalabmessungen und den kinematischen Eigenschaften des RBG wider. Mit dem Modell können also auch ungewöhnliche Regalabmessungen genau berechnet werden, bei denen $b$ ungleich 1 ist und die Synchronfahrgerade ${ }^{4}$ nicht mehr die obere Ecke der Regalwand schneidet. Bei diesen Konfigurationen stößt das Modell der repräsentativen Punkte an seine Grenzen und liefert ungenauere Ergebnisse. Ein Nachteil des Fahrwegmodells ist, dass es keine Beschleunigungszeiten berücksichtigt. Das vorgestellte Modell wird deshalb von Chang et al. [Cha95] und Hwang et al. [Hwa90] erweitert. Für die genaue Kalkulation der Einfahrzeit hat sich das Modell nach Chang als sehr gut erwiesen. Dieses Modell legt anstatt reiner Trapezfahrten ein korrektes Geschwindigkeitsprofil zugrunde und erzielt damit sehr genaue Ergebnisse. In Abbildung 3 links werden die Modelle nach Chang und nach FEM 9.851 gegenübergestellt und mit den Werten einer Monte Carlo Simulation (MC Simulation) verglichen. Das Modell nach Bozer und White bzw. nach Chang liefert auch für extreme Regalabmessungen sehr genaue Ergebnisse, während das nach der FEM 9.851 nur im Bereich zwischen $0,5 \leq b \leq 2$ gültig ist. Im Normalfall ist dieser Anwendungsbereich ausreichend, für eine automatische Dimensionierung des Lagers müssen jedoch auch extreme Verhältnisse zwischen RBG-Geschwindigkeiten und Regalabmessungen berechnet und miteinander verglichen werden.

Auch im Falle einer Verschiebung des Übergabepunkts an der Regalstirnseite liefert ersteres Modell genauere Ergebnisse (vgl. Abbildung 3 rechts und [Lip03]).
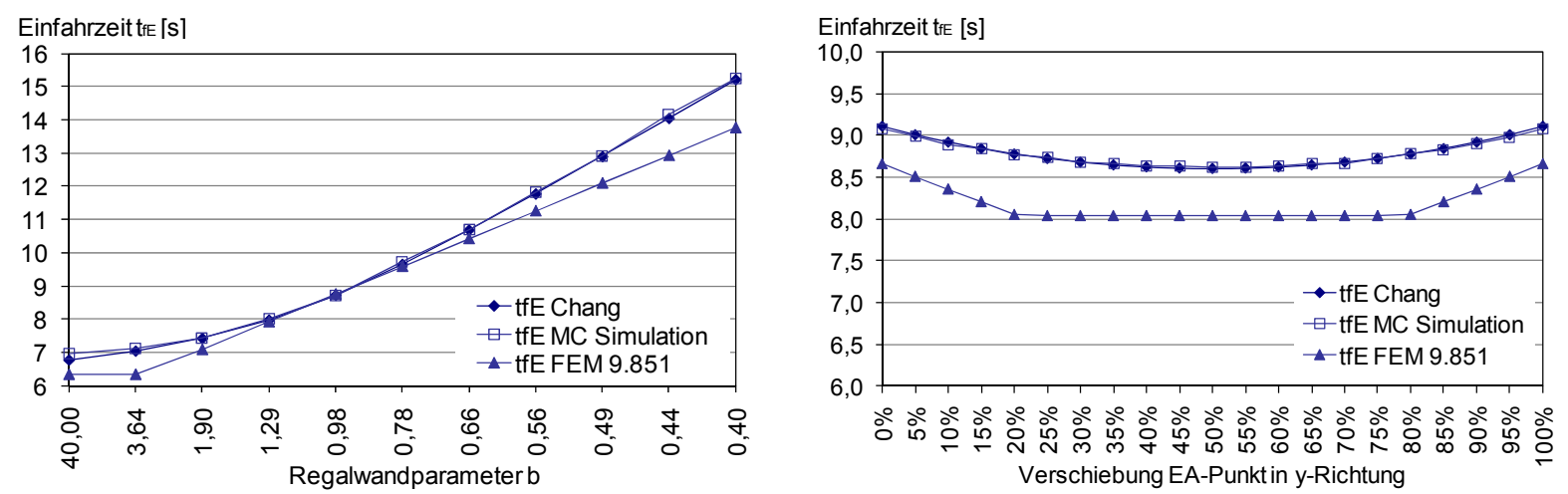

Abbildung 3: Vergleich der berechneten mittleren Einfahrzeit verschiedener Modelle. Links: bei unterschiedlichen Regalabmessungen; Rechts: Bei einer Verschiebung des Übergabepunkts an der Regalstirnseite

Für die Querfahrzeiten hat es sich als zielführender erwiesen, zu den ermittelten durchschnittlichen Zeiten eine Bremsbeschleunigungskonstante $t_{a} z u$ addieren, wie schon von Gudehus hergeleitet [Gud72].

\footnotetext{
${ }^{4}$ Gerade, die vom LAM bei Start in einer Regalecke und bei maximaler Beschleunigung in $x$ - und y-Richtung durchlaufen wird.
} 
Beim Einsatz mehrerer Lastaufnahmemittel oder bei speziellen Auslagerstrategien ist es mit dem RBG möglich, bei der Anfahrt aus mehreren Auslagerfächern auszuwählen. Um möglichst kurze Wege zu fahren, wird eine Fahrwegoptimerung durchgeführt und das nächstgelegenste Auslagerfach angefahren. Die mittlere Querfahrtszeit verkürzt sich, je größer die Anzahl $n$ der potentiellen Anfahrlagerfächer ist. Um diesem Umstand Rechnung zu tragen, wird das Modell erweitert und die mittlere Einfahrzeit und Querfahrzeit für $n$ beliebige Anfahrtspunkte hergeleitet. Auch bei diesen Spielzeitkomponenten kann eine ausreichend genaue Übereinstimmung zur Monte Carlo Simulation nachgewiesen werden.

\section{Stochastisches Umgebungsmodell}

Die Einlagerstrategie Einlagerung nahe Auslagerung wird in der Praxis sehr häufig bei sowohl einfachtiefen als auch doppeltiefen Lagern eingesetzt. Der Fahrweg, der dabei zwischen dem Einlagerfach und dem Auslagerfach anfällt, ist im Wesentlichen nur von den kinematischen Eigenschaften des RBG, den Lagerfachabmessungen und vom Lagerfüllgrad abhängig. Je voller das Lager, desto weiter entfernt ist im Mittel das Einlagerfach. Um die dafür benötigte Fahrzeit abzuschätzen, wird das in Abbildung 4 skizzierte Umgebungsmodell [See06] eingesetzt.
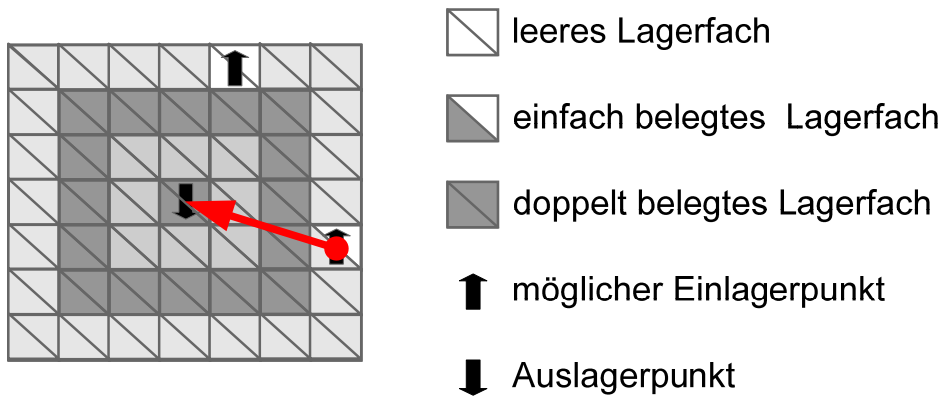

Abbildung 4: Umgebungsmodell zur numerischen Approximation der mittleren Fahrzeiten zu den nächstgelegenen leeren Lagerfächern

Das Modell umfasst einen Regalwandabschnitt mit kartesischen Lagerplatzkoordinaten. In einem ersten Schritt wird die Wahrscheinlichkeit berechnet, dass ein leeres Lagerfach in einem bestimmten Abstand vom Auslagerfach frei ist. Lagerfächer mit demselben Fachabstand bilden eine Umgebung und sind in der Abbildung farblich einheitlich hinterlegt. Die Abbildung zeigt nur eine Regalwand. Dem Auslagerfach gegenüberliegend befindet sich die zweite Regalwand, in der die Einlagerung ebenfalls stattfinden kann. Die nullte Umgebung besteht demnach aus dem dem Auslagerfach direkt gegenüberliegenden Lagerfach, die erste Umgebung aus den 16 Lagerfächern, die den Abstand 1 zum Auslagerfach haben, etc. Die Anfahrwahrscheinlichkeiten hängen von der Lagerfüllung und den Belegungseigenschaften des Regals ab. Je größer der Füllgrad, desto wahrscheinlicher wird es, dass sich kein leeres Fach innerhalb der betrachteten Umgebungen befindet, und desto ungenauer wird das Modell, wenn zu wenige Umgebungen betrachtet werden. In einem zweiten Schritt wird die Fahrzeit zu jedem Lagerfach der Umgebung berechnet und mit seiner (c) 2011 Logistics Journal : Proceedings - ISSN 2192-9084 
Anfahrwahrscheinlichkeit gewichtet. Die Summe ergibt die mittlere Fahrzeit zwischen den beiden Fächern.

Durch leichte Abwandlungen des Modells lassen sich die Querfahrzeiten für die Einlagerungen im einfachtiefen Lager und Umlagerungen im doppeltiefen Lager und/oder mit doppelbreitem LAM berechnen.

Die Näherung mittels dieser numerischen Approximation deckt sich gut mit den simulierten Werten. Signifikante Abweichungen treten erst bei wenig relevanten Lagerfüllgraden größer 95\% auf.

\section{Deterministische Modelle}

Neben den bereits beschriebenen Spielzeitkomponenten, die den Mittelwert stochastisch verteilter Fahrzeiten abbilden, gibt es eine Reihe von Spielzeitkomponenten, welche deterministisch ermittelt werden können.

Die Auf- und Abgabe der Ladeeinheiten durch das LAM verursacht z. B. einen konstanten Zeitbedarf. Dieser setzt sich i.d.R. aus der Hub- und Senkzeit sowie der Fahrzeit des LAM zusammen und ist von der Geschwindigkeit, der Beschleunigung und der zu überbrückenden Stecke abhängig. Auf die Zykluszeit für die Ein- oder Auslagerung einer Ladeeinheit haben neben den Eigenschaften des LAM die Lagertiefe und die Übergabestrategie am Übergabepunkt Einfluss.

Unmittelbar nach einer Fachanfahrt ist es notwendig, den Mast des RBG ausschwingen zu lassen und das LAM für die Ein- oder Auslagerung exakt auszurichten. Die Mastausschwingzeit überwiegt dabei alle restlichen Einflussgrößen. Sie hängt wesentlich von der Arbeitshöhe des LAM ab und ist nicht über die gesamte Regalhöhe konstant. Je größer die Höhe ist, in welcher sich das LAM mit der Ladeeinheit bewegt, desto größere Schwingungen treten auf und desto mehr Beruhigungszeit fällt an. Von RBG sind häufig die Mastausschwingzeiten bei minimaler und maximaler Höhe des LAM bekannt. In erster Näherung werden die Werte für die Zwischenhöhen durch eine lineare Interpolation berechnet.

Bewegungen des RBG werden von Sensorik und Steuerungseinheiten ausgelöst und gesteuert. Durch Schalt- und Kontrollvorgänge treten deshalb bei jeder RBG- und LAM-Bewegung Totzeiten auf. Die Totzeiten sind unterschiedlich lang, werden in der Praxis aber durch eine Konstante ausreichend genau angenähert [Gud72].

Ebenfalls einfach zu berechnen sind die Zwischenfahrzeiten zwischen den Übergabepunkten. Diese Zwischenfahrten werden notwendig, wenn der Aufnahmeund Abgabepunkt (ÜE und ÜA) getrennt sind und nicht mehr auf einer Höhe gegenüberliegen. Die Fahrten zwischen diesen Punkten können über eine normale Fahrzeitberechnung unter Berücksichtigung der Tchebychevfahrt (parallele Fahrt des Fahr- und Hubwerks) berechnet werden. 


\subsection{Berechnung der Spielzeit einer Lagergasse}

Für die Umschlagsleistungsbestimmung eines Hochregallagers sind drei Fälle zu unterscheiden, die je nach Einsatzgebiet unterschiedlich wichtig sind. Die Maximalleistung eines Lagersystems wird meistens für kombinierte Arbeitsspiele, d.h. für Spiele, in denen Ein- und Auslagerungen kombiniert durchgeführt werden, angegeben. Die Kenngröße ist dabei die Anzahl der Ladeeinheiten pro Zeiteinheit, die ein- oder ausgelagert werden. Für gewisse Anwendungsfälle ist aber nicht die kombinierte Leistung ausschlaggebend, sondern die Umschlagsleistung in Spitzenzeiten, in denen reine Einlagerungen (z. B. Einlagerung von angelieferter Ware zu einer bestimmten Tageszeit) oder reine Auslagerungen (z. B. schnelle Auslagerung von Kundenbestellungen zu einer bestimmten Tageszeit) gefahren werden. Das Modell ist dafür ausgelegt, alle drei Fälle abzudecken und erlaubt es auch, vermischte Grenzleistungen zu berechnen.

\section{Berechnung des Spielanteils für die Ein- und Auslagerung (a)}

Die Spielzeit einer Regalgasse setzt sich aus den beiden Spielanteilen (a) und (b) zusammen. Im ersten Schritt wird der Spielanteil (a) berechnet. Dieser beginnt beim ersten Ein- oder Auslagerfach im Regal und bildet das gesamte Spiel im Regal bis zur letzten Ein- oder Auslagerung ab. Die Zusammensetzung des Spielanteils hängt neben der Lagerkonfiguration und -strategie auch von der Spielart ab (Einlagerung, Auslagerung oder kombiniertes Spiel). Im Fall eines einfachtiefen Lagers mit einem LAM und der Einlagerstrategie zufällige Einlagerung besteht das kombinierte Spiel (in diesem Fall Doppelspiel) aus einer LAM-Zykluszeit für die Einlagerung, einer Querfahrt (beliebiges Fach im Regal zu einem anderen beliebigen Fach im Regal) und einer abschließenden Zykluszeit für die Auslagerung. Der Spielanteil (a) nicht kombinierter Spiele besteht lediglich aus einer einzelnen Einlagerung bzw. Auslagerung. Für alle verwendeten Spielzeitkomponenten müssen zusätzlich die anteilige Totzeit und eventuell die Mastausschwingzeit mit eingerechnet werden.

In Abbildung 5 sind zwei Beispiele für komplexere Lagerkonfigurationen skizziert. Die linke Skizze zeigt ein einfachtiefes Lager mit drei LAM und der Strategie Einlagerung nahe Auslagerung. Spielanteil (a) für das kombinierte Spiel besteht aus einer Einlagerung in der Nähe des Auslagerfachs, einer Fahrt zu diesem, der Auslagerung und der Fahrt zu einem leeren Fach in der Nähe des nächsten Auslagerfachs, in welchem die Einlagerung stattfinden kann. Diese Vorgänge wiederholen sich zwei weitere Male, bis alle drei LAM inre Ladeeinheiten eingelagert und eine andere Ladeeinheit ausgelagert haben. Der Spielanteil besteht also aus drei Querfahrten vom nächstgelegenen Einlagerfach zum Auslagerfach, sechs einfachtiefen LAMZykluszeiten und zwei Querfahrten zwischen dem Auslagerfach und dem nächsten Einlagerfach. Nach der ersten Einlagerung kann bei einer Reihenfolgeoptimierung aus zwei Einlagerfächern ausgewählt werden. Es kommen für diesen Fall verkürzte Querfahrzeiten zur Anwendung. 
Die rechte Skizze in Abbildung 5 zeigt ein doppeltiefes Lager mit einem LAM und der Strategie Umlagerung nahe Auslagerung. Das Spiel beginnt mit einer Einlagerung, die abhängig von der Kanalbelegung in das vordere oder hintere Fach erfolgt. Lippolt [Lip03] entwickelte ein System zur Berechnung der Wahrscheinlichkeiten im doppeltiefen Lager. Damit kann in Abhängigkeit des Lagerfüllgrads abgeschätzt werden, wie wahrscheinlich die Einlagerung in das hintere bzw. vordere Fach oder wie wahrscheinlich die Notwendigkeit einer Umlagerung ist. Nach der Einlagerung erfolgt eine Querfahrt zum Auslagerfach. Mit der Wahrscheinlichkeit P1 kann die Ladeeinheit vorne oder mit der Wahrscheinlichkeit P2 direkt vom hinteren Fach ausgelagert werden. In beiden Fällen ist das Spiel beendet. Mit der Wahrscheinlichkeit 1-(P1+P2) muss zuerst eine Umlagerung getätigt werden. Die vordere Ladeeinheit wird dazu ausgelagert und es erfolgt eine Querfahrt zum nächstgelegenen Lagerkanal mit mindestens einem leeren Lagerfach. Die Ladeeinheit wird darin eingelagert und es erfolgt die Rückfahrt. Nun kann die eigentlich erwünschte Auslagerung getätigt werden.
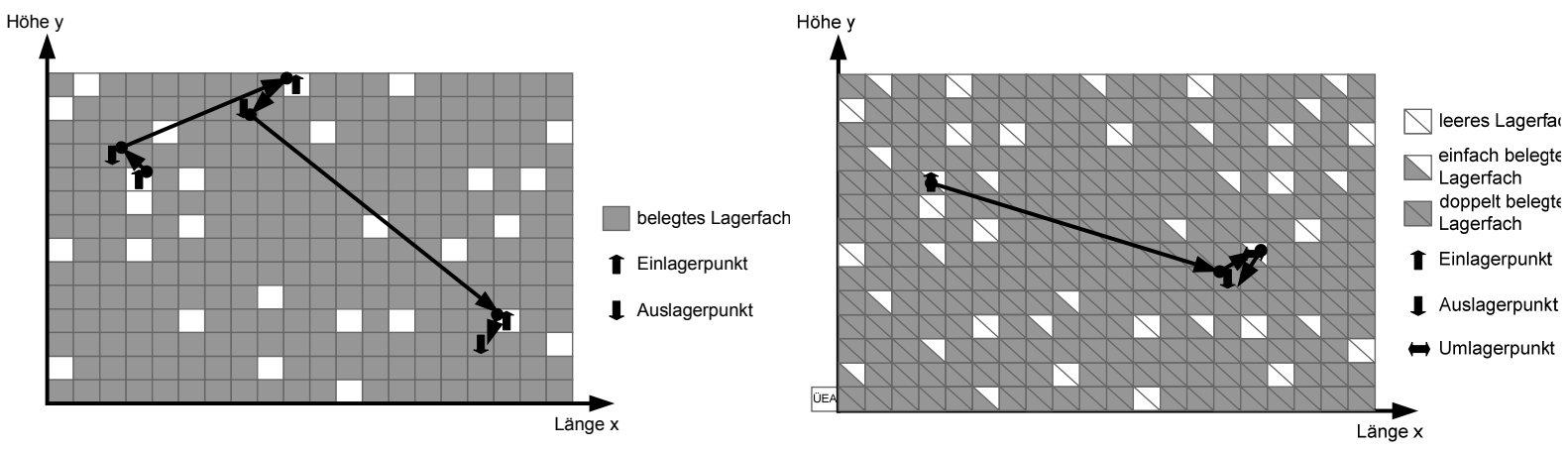

Abbildung 5: Skizze Spielanteil (a). Links: Einfachtiefes Lager mit drei LAM und der Strategie „Einlagerung nahe Auslagerung“; Rechts: Doppeltiefes Lager mit einem LAM und der Strategie „Umlagerung nahe Auslagerung“

Für das Rechenwerkzeug werden über 100 sinnvolle Spielanteile (a) für unterschiedliche Lagerkonfigurationen und Lagerstrategien definiert.

\section{Berechnung des Spielanteils für die Aufnahme und Abgabe (b)}

Zur Komplettierung eines Arbeitspiels muss noch der Spielanteil (b) berechnet werden. Dieser Spielanteil beinhaltet die Auf- und Abgabe am Übergabepunkt und die Ein- und Ausfahrt aus dem Regal. Auf diesen Spielanteil haben die Lage der Übergabepunkte und die Intensität der darüber führenden Materialflüsse sowie die Ruhepositionsstrategie wesentlichen Einfluss. Die Ruhepositionsstrategie beschreibt das Verhalten des RBG nach erfolgtem Abschluss eines Spiels. Bei einer Auslagerung oder einem kombinierten Spiel, ist das Arbeitsspiel nach der Abgabe am Übergabepunkt abgeschlossen. Nach einer reinen Einlagerung besteht die Möglichkeit, im Regal zu verharren, um dann bei einer eventuell folgenden Auslagerung schon näher am Auslagerfach zu sein. Folgen eine Einlagerung oder ein kombiniertes Spiel, muss das RBG zuerst zum Übergabepunkt ausfahren. Aus 
der Intensität der Materialflüsse über die Übergabepunkte werden die Wahrscheinlichkeiten berechnet, an welchen Punkten ein Arbeitsspiel endet bzw. startet.

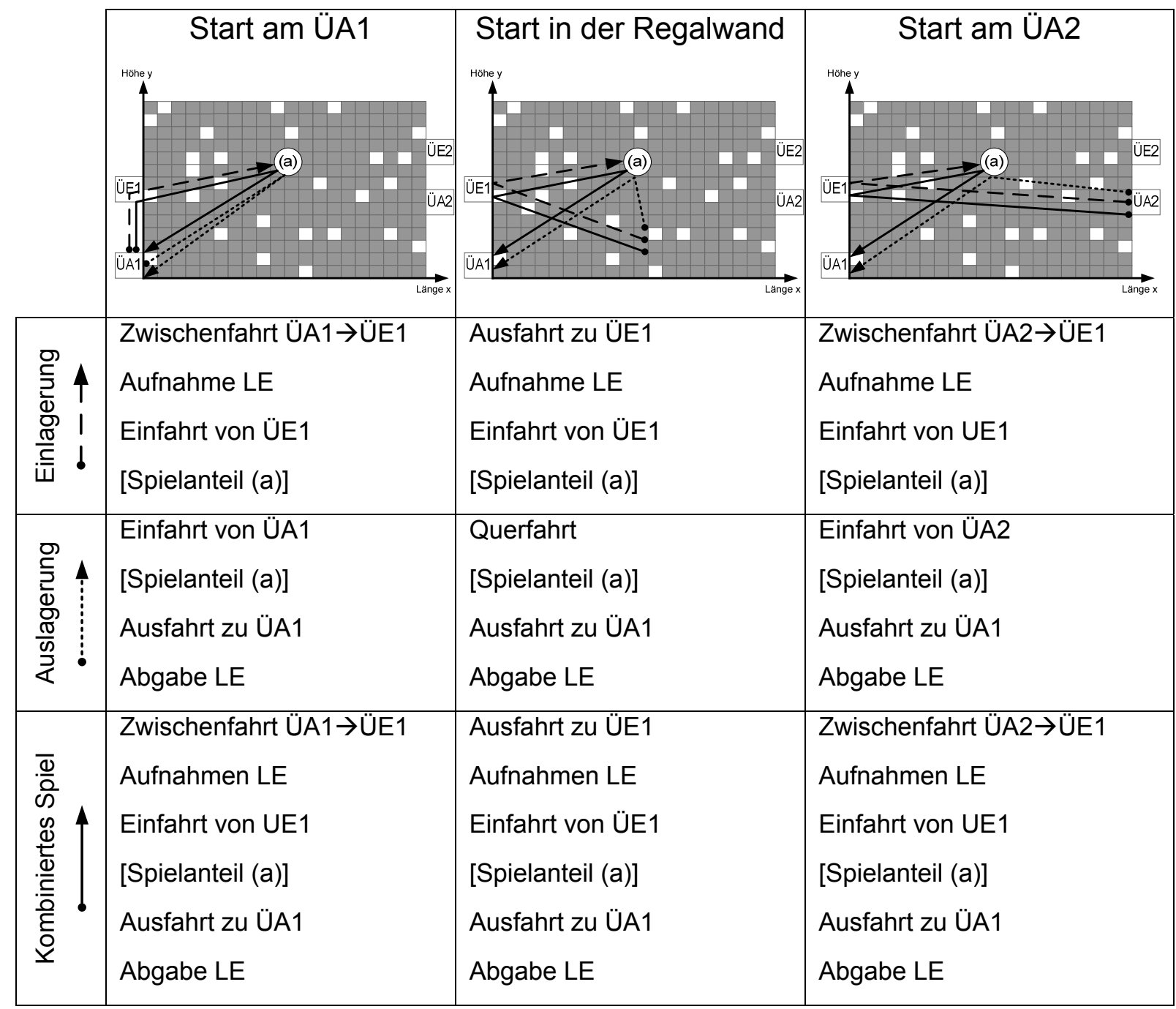

Abbildung 6: Spielanteile (b) für Einlagerung, Auslagerung und kombiniertes Spiel bei unterschiedlichen Startpunkten; (a)=Übergang zum Spielanteil (a)

Innerhalb des Berechnungsmodells wird ein Gleichungssystem verwendet, welches bis zu zwei Aufnahme- und Abgabepunkte abdecken kann. Dieses Gleichungssystem geht von einer Gleichverteilung der Ein- und Auslageraufträge in der Belastungsspitze aus. Bei der beschriebenen Ruhepositionsstrategie und vier Übergabepunkten sind drei Startpunkte eines Arbeitsspiels möglich: Der erste Abgabepunkt ÜA1 (nach Auslagerung oder kombiniertem Spiel), der zweite Abgabepunkt ÜA2 (nach einer Auslagerung oder kombiniertem Spiel über den zweiten Abgabepunkt) und im Regal selbst (nach erfolgter Einlagerung). Ein Arbeitsspiel kann von allen drei Startpunkten aus beginnen. Zur Berechnung des Spielanteil (b) werden die Spielzeitkomponenten für die Aufnahme, die Einfahrt zum Einlagerpunkt und dann (nach dem Spielanteil (a)) die Ausfahrt und Abgabe summiert und mit der entsprechenden Auftrittswahrscheinlichkeit gewichtet. 
Abbildung 6 zeigt die Spielanteile (b), die bei Einlagerung, Auslagerung und kombiniertem Spiel mit den drei unterschiedlichen Startpunkten auftreten.

Bei der Einlagerung muss zuerst eine Ladeeinheit vom ÜA1 abgeholt werden. Entsprechend dem Startpunkt wird dazu eine Zwischenfahrt vom ÜA1 oder ÜA2 notwendig. Entspricht ÜA1 dem ÜE1, so entfällt die Zwischenfahrt. Ist der Startpunkt im Regal, wird eine Ausfahrt durchgeführt. Nach der Aufnahme der Ladeeinheit (oder bei Mehrfachlastaufnahmemittel mehrerer Ladeeinheiten) erfolgt die Einfahrt in das Regal. Damit ist der Spielanteil abgeschlossen. Zur Komplettierung des Arbeitsspiels muss noch der Spielzeitanteil (a) ausgeführt werden, welcher die Einlagerungen und Querfahrten beinhaltet. Die Zusammensetzung der Spielanteile (b) für die Auslagerspiele und die kombinierten Spiele können der Abbildung 6 entnommen werden.

\subsection{Berechnung der Umschlagsleistung eines Lagers}

Um die mittlere Spieldauer für die Einlagerung, Auslagerung und das kombinierte Spiel zu berechnen, werden der Spielanteil (a) und der gewichtete Spielanteil (b) zusammengezählt. Unter der Annahme von unabhängigen Lagergassen kann die Umschlagsleistung des gesamten Lagers durch die Skalierung der Leistung einer einzelnen Gasse erfolgen. Durch die Verkettung in der Lagervorzone entsteht jedoch eine Abhängigkeit zwischen den Gassen. Wird beispielsweile eine sequenzierte Auslagerung über das gesamte Lager angestrebt, entstehen Wartezeiten durch blockierte RBG. Lee [Lee97] und Hur [Hur04] entwickelten dafür dynamische Warteschlangenmodelle, die bei der Berechnung einfacher Lagerarten angewendet werden können. Die analytische Berechnung ist aber komplex und rechenintensiv. Deshalb werden Parameterstudien mit einer eigens entwickelten Ablaufsimulation durchgeführt, um den Einfluss verketteter Gassen abschätzen zu können. Es werden Abschlagswerte für die Umschlagsleistung ermittelt, die von der Sequenzstrategie und der Gassenanzahl abhängen.

Für die Auslegung von Lagersystemen und den Vergleich unterschiedlicher Lagerkonfigurationen ist es zielführend, die Auslastung des Systems zu berechnen (Auslastungsgrad $\rho=T_{\text {benötigt }} / T_{\text {verfügbar }}$ ).

\subsection{Ergebnis}

Das vorgestellte Lagermodell kann die Umschlagsleistung von unterschiedlichen automatischen Lagersystemen berechnen. Es werden einfach- und doppeltiefe Lager mit ein bis drei LAM, die einfach- oder doppelbreit ausgeführt sind, abgedeckt. Es können mehrere Übergabepunkte sowie eine Verschiebung derer mit einbezogen werden. Das Modell berücksichtigt unterschiedliche Ein-, Um- und Auslagerstrategien. Eine Fahrwegoptimierung kann ebenso wie die Ruhepositionsstrategie und die Sequenzierung der ausgelagerten Ladeeinheiten eingerechnet werden. 


\section{$4 \quad$ Kostenberechnung}

Die Thematik der wirtschaftlichen Auslegung automatischer Lagersysteme war in der Vergangenheit im Vergleich zur Durchsatzberechnung weit weniger Gegenstand wissenschaftlicher Betrachtung. Ansätze dazu stellen Gudehus [Gud73a] [Gud73b] und Fröhlich [Frö80] vor. Bei der entwickelten Planungsvorgehensweise ist eine gute Kostenschätzung die Voraussetzung für gute Ergebnisse. Können die Kosten nicht hinreichend genau geschätzt werden, erfolgt eine ungenaue Dimensionierung. Fließen Parameter falsch ein, kann die Optimierung in eine ungünstige Richtung verlaufen und den Vergleich von Planungsalternativen verzerren.

Zur Kostenschätzung wird das Bottom-Up-Verfahren angewendet. Dieses Verfahren schätzt die Kosten über die Summe aller Einzelteile (hier die Lagerkomponenten). Es ermöglicht eine detaillierte Schätzung, welche beim Variantenvergleich ihre Aussagekraft beibehält. Die Basis des Verfahrens ist eine Datensammlung zu den Lagerkomponenten, welche zusammen mit Projektpartnern aus der Industrie erarbeitet und in einer Datenbank strukturiert wird. Diese enthält neben den technischen Angaben zur Geometrie alle relevanten Größen für die Leistungsberechnung sowie die zugehörigen Kostensätze.

Für die Kostenschätzung wird die Systemgrenze zwischen Hochregallager und Lagervorzone gezogen. Gegenstand der Betrachtung ist demnach das Lagersystem an sich, das umgebende Gebäude, die Fördertechnik, welche die Lagergassen an die Lagervorzone anschließt, und die IT-Technik, welche zum Betrieb des Lagersystems notwendig ist. Die Komponenten werden in die Kategorien Gebäude, Lagertechnik und Informationstechnik untergliedert.

\subsection{Gebäude}

Der Investitionsaufwand für das Gebäude setzt sich aus Preisen für das Grundstück, die Bodenplatte, die Wände und das Dach zusammen. Kostentreiber sind die jeweils verbauten Flächen und die thermische Auslegung des Lagers. Bei der Ausführungsform der Bodenplatte ist zusätzlich die benötigte Traglast entscheidend. In den Wandpreis fließt auch die realisierte Bauhöhe mit ein.

Der Grundstückspreis wird nicht über vorgegebene Datensätze berechnet. Enorme Unterschiede des Preisniveaus in den unterschiedlichen Bundesländern machen eine sinnvolle Abschätzung unmöglich. Der Grundstückspreis fließt deshalb als Planungsvorgabe in die Kostenberechnung mit ein.

\subsection{Lagertechnik}

Die Kosten der Lagertechnik setzen sich aus den Kosten für Regal, Regalbediengeräte, Brandschutz, Übergabepunkte und sonstige Fördertechnik zusammen. 
Für die Kostenschätzung des Regals wird ein Verfahren aus der Industrie übernommen. Dort werden die Regalkosten häufig über einen Kostensatz pro Stellplatz geschätzt. Dieser ist von der Stellplatzlast, der Größe der Ladeeinheiten und der realisierten Höhe abhängig. Kostenvorteile ergeben sich bei doppeltiefen Regalen. Alternativ zu diesem praxisnahen Ansatz wird ein Verfahren entwickelt, welches in grober Näherung eine statische Auslegung des Regalsystems durchführt, und über die Masse und Qualität an verbautem Stahl den Investitionsaufwand schätzt. Bei der Dimensionierung der Dicke der Palettenträger ist die Traglast und die Fachlänge entscheidend. Die maximale Durchbiegung muss innerhalb des Toleranzbereichs des RBG liegen. Für die Stützen werden die Traglast (Gewicht der Ladeeinheiten und der Palettenträger), der Diagonalenabstand und die Seitenkräfte wie Windlast und Stöße des RBG beachtet (Abbildung 7).

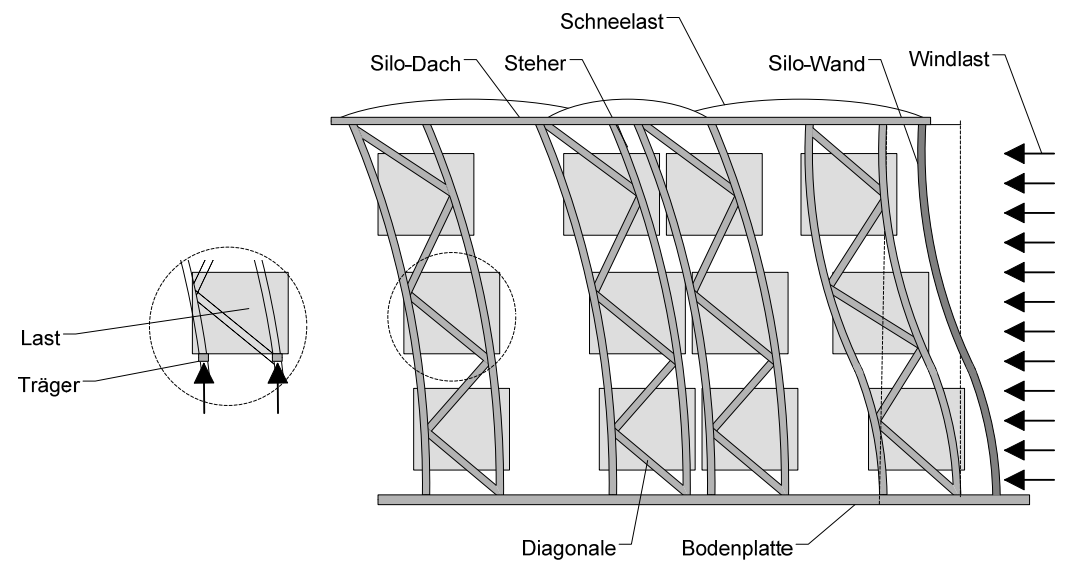

Abbildung 7: Skizze der Einflüsse auf die Dimensionierung eines Regalsystems bei der Auslegung der Steher

Zur Kostenschätzung der Regalbediengeräte werden diese basierend auf einer Marktanalyse [NN10] klassifiziert. Die Klassen unterscheiden sich in maximaler Bedienhöhe, Traglast und Ladeeinheitengröße. Die RBG können mit unterschiedlichen LAM kombiniert werden, um die unterschiedlichen Lagerkonfigurationen zu bedienen. Zusammen mit einem namhaften Hersteller können die Marktpreise für typische RBG der erarbeiteten Klassen und die zugehörigen technischen Eigenschaften wie Beschleunigungen, Geschwindigkeiten, LAM-Zykluszeiten etc. ermittelt werden. Insgesamt liegen dem Rechenwerkzeug 34 Datensäte für unterschiedliche RBG vor, die jeweils an die 40 Informationen beinhalten. Zu der bestehenden Datensammlung können beliebig viele weitere RBG hinzugefügt werden, welche auch nicht den Klassenvorgaben entsprechen müssen.

Der Investitionsaufwand für die Übergabepunkte ist weitestgehend vom verwendeten LAM abhängig. Bei der Verwendung von mehreren LAM werden an diesen Punkten entsprechend viele Ladeeinheiten parallel übergeben. Bei einem doppelbreiten LAM kommt häufig ein doppelbreiter Übergabepunkt zum Einsatz. Die Übergabepunkte sind über Fördertechnik an die Lagervorzone angebunden. Diese Fördertechnik 
übernimmt eine puffernde Funktion. Die Kosten dafür hängen im Wesentlichen von der Anzahl an verbauten Antrieben und damit mit der Pufferlänge zusammen.

Der Brandschutz wird in den meisten Lagern durch eine Sprinkleranlage gewährleistet. Hier werden die Kosten über die Stellplatzanzahl und die Bauhöhe abgeschätzt.

\subsection{IT-System}

Der Investitionsaufwandsschätzung für das IT-System liegen die Kosten der einzelnen Module eines Lagerverwaltungssystems zugrunde. Je nach Lagerkonfiguation und -strategien sowie der Größe des Lagers werden unterschiedliche Module benötigt, so dass der Investitionsaufwand variiert.

\subsection{Laufende Kosten}

Zur Berechnung der laufenden Kosten werden für die verbauten Kostengruppen prozentuale Aufschlagswerte auf den Investitionsaufwand bestimmt. Über diese Werte werden die Wartungs- und die Energiekosten des automatischen Lagersystems angenähert.

\subsection{Ergebnis}

Mit der beschriebenen Methodik werden die Investitionskosten des Gesamtsystems ermittelt. Die Datenbasis der Methodik umfasst Datensätze zu Palettenhochregallagern als auch Automatischen Kleinteilelagern in unterschiedlichen Größen und Ausprägungsformen (z. B. Silo- und Hallenbauweise). Durch die Einbeziehung der laufenden Kosten werden die Kosten über einen bestimmten Zeitraum berechnet und auf Basis des effektiven Nutzungszeitraums eine kalkulatorische Kostenrechnung durchgeführt.

\section{Implementierung eines Rechenwerkzeugs zur integrierten Lagersystemplanung}

Die Modelle zur Umschlagsleistungs- und Kostenberechnung werden in einem datenbankgestütztem Rechenwerkzeug zusammengeführt. Dieser Demonstrator ermöglicht die parallele Betrachtung der beiden Größen und eine mathematische Optimierung zur Dimensionierung von Planungsvarianten. Diese werden mit anderen Lagervarianten, die sich in Lagerkonfiguration und Betriebsstrategie unterscheiden, verglichen.

\subsection{Aufbau des Planungswerkzeugs}

Das Rechenwerkzeug ist mit der Programmiersprache $\mathrm{C \#}$ in der Entwicklungsumgebung MS Visual Studio implementiert. Die Oberfläche des Hauptfensters gliedert sich in zwei Bereiche (Abbildung 8 links). Im linken Bereich werden die planungsrelevanten Daten, die für sämtliche Planungsvarianten gültig 
sind, dargestellt und eingegeben. Im rechten Bereich werden unterschiedliche Planungsvarianten angelegt und miteinander verglichen.

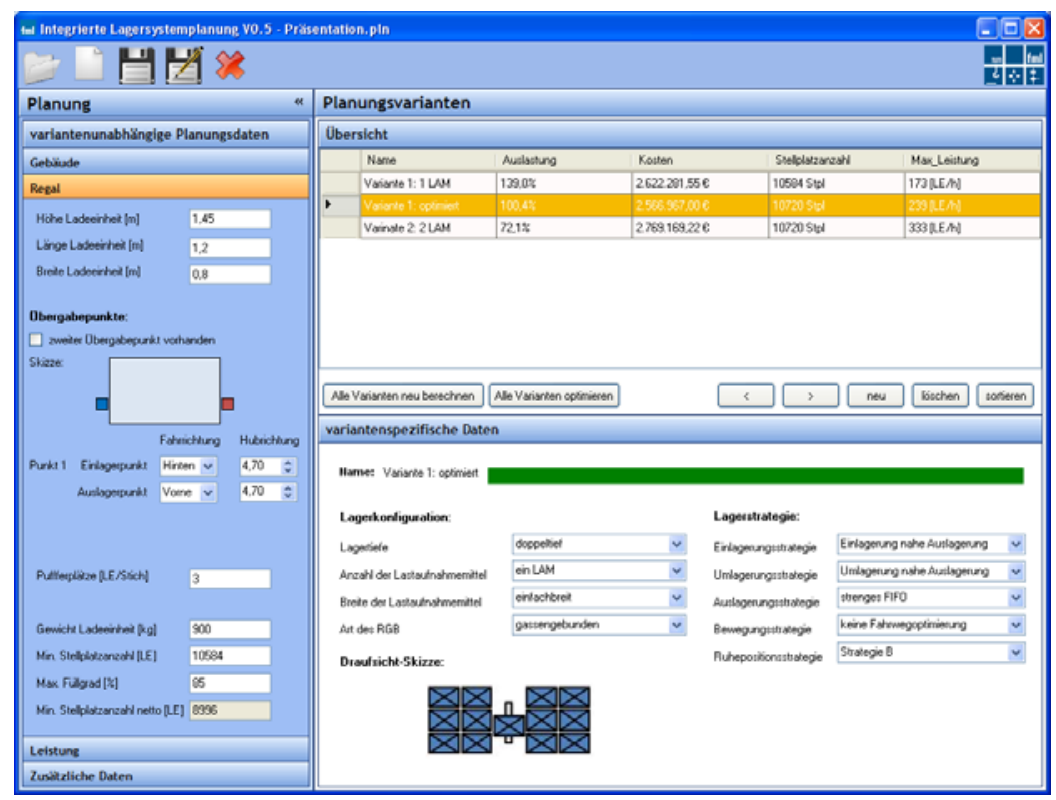

Abbildung 8: Screenshot des implementierten Planungswerkzeugs. Hauptfenster für die Definition der Planungsdaten und Planungsvarianten

Die Planungsdaten auf denen sich die Berechnungsmodelle beziehen, werden über eine Eingabemaske eingegeben. Sie beinhalten die Gebäudedaten, wie der maximal zur Verfügung stehenden Länge, Höhe und Breite, die Abmessungen der zu lagernden Ladeeinheiten, die minimale Stellplatzanzahl, die Lage der Übergabepunkte und die Materialflusse über die Übergabepunkte. Zusätzlich können Eingaben getroffen werden, ob und welche Art der Sequenzierung im Lager vorgenommen werden muss und ob eine Brandschutzanlage in das Lager integriert werden soll. Alle Planungsvarianten beziehen sich auf diese Planungsdaten. Die Varianten unterscheiden sich in Lagerkonfiguration, -strategie oder in ihrer geometrischen Dimensionierung. Sie werden tabellarisch aufgelistet und können über die Parameter Auslastung, Investitionskosten, Stellplatzanzahl und maximale Umschlagsleistung miteinander verglichen werden.

Jede Planungsvariante kann für sich genauer dimensioniert und betrachtet werden. Neben den geometrischen Abmessungen (Regallänge und -höhe, Anzahl der Gassen, etc.) kann aus einer Liste ein für die Planungsvariante geeignetes RBG oder die automatische Verwendung des am besten geeigneten RBG ausgewählt werden. Im Hintergrund werden parallel zu den Benutzereingaben die exakte Lagergeometrie und die realisierbare Stellplatzanzahl sowie Umschlagsleistung und Investitionsaufwand berechnet. Die Berechnung greift dabei auf eine Datenbank zu, in welcher die Kosten- und Leistungsdaten sowie die geometrischen Eigenschaften der einzelnen Komponenten des Lagers hinterlegt sind (vgl. Kapitel 4). In einem weiteren Fenster werden Zwischenschritte und detaillierte Informationen zu den Berechnungen ausgegeben. Optional können ein Vergleich mit einer Monte Carlo 
Simulation und eine Optimierung der Lagerdimensionierung durchgeführt werden. Mittels Sensitivitätsanalysen können die Auswirkungen von Änderungen der Planungsdaten auf ein optimal ausgelegtes Lager untersucht und visualisiert werden.

\subsection{Optimierung einer Planungsvariante}

Für die Optimierung der Planungsvarianten können unterschiedliche Optimierungsalgorithmen eingesetzt werden. Das vorliegende Optimierungsproblem bezieht sich auf eine nichtlineare unstetige Funktion [Alt02]. Die Veränderlichen, hier als der Vektor $\mathbf{x}$ dargestellt, sind beispielsweise die Lagerlänge und -breite. Aus der Anzahl der Parameter ergibt sich die Dimension des Problems. In diesem Fall liegt ein mehrdimensionales Optimierungsproblem vor, das folgendermaßen lautet:

$$
\min _{x \in X} f_{\text {Kosten }}(x) \quad f_{\text {Kosten }}: \mathbb{R}^{\mathrm{n}} \rightarrow \mathbb{R} \quad X \subseteq \mathbb{R}^{\mathrm{n}}
$$

Unter Einhaltung der Restriktionen:

$$
\text { NB1: } f_{\text {Leistuna }}(\boldsymbol{x}) \geq \text { geforderte Leistung }
$$

NB2: $f_{\text {Stellplatzanzahl }}(\boldsymbol{x}) \geq$ geforderte Stellplatzanzahl

$$
\text { NB3: } f_{\text {Laenae }}(\boldsymbol{x}) \leq \text { maximale Laenge }
$$

$N B 4: \ldots$

Die Zielfunktion der Optimierung ist i.d.R. der Betrag der Investitionskosten, sie kann aber auch der Betrag des Verhältnisses der

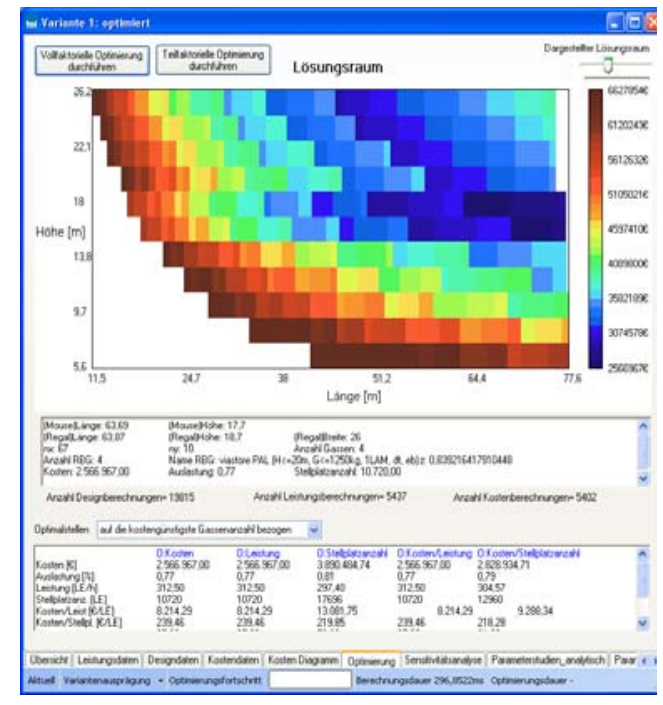

Abbildung 9: Screenshot der graphischen Visualisierung des Lösungsraums und der Ausgabe der Optimalstellen Kosten zur Umschlagsleistung oder zur Stellplatzanzahl sein. Die Nebenbedingungen sind die Umschlagsleistung, die Stellplatzanzahl, die Abmessungen der Lagerhalle etc. Für eine gültige Planungsvariante müssen sämtliche Nebenbedingungen, die sich aus den Planungsvorgaben ergeben, erfüllt sein.

Unter anderem durch die Notwendigkeit der Verwendung diskreter Parametern erzielen verschiedene Optimierungsalgorithmen keine zufriedenstellenden Ergebnisse im Vergleich mit der vollfaktoriellen Suche. Bei dieser Methode werden sämtliche Parameterkombinationen durchgetestet. Um die Anzahl der Funktionsauswertungen zu reduzieren, wird ein Branch and Bound Verfahren eingesetzt. Mit Hilfe dieses Verfahrens können Teiläste der Optimierung verworfen und Rechenzeit eingespart werden. Die Teiläste werden nicht mehr 
betrachtet, wenn sie sicher suboptimal sind. Eine solche Schlussfolgerung kann beispielsweise bei einer Parameterkonfiguration mit einem teureren und gleichzeitig langsameren RBG als dem momentan Optimalen gezogen werden. Weitere Rechenzeit wird durch eine schrittweise Berechnung der Nebenbedingungen eingespart. Bei Nichterfüllung einer Bedingung wird die Parameterkonfiguration verworfen und nicht weiter betrachtet. Die vollfaktorielle Suche wird auf dem verwendeten Testrechner im Zehntelsekundenbereich durchgeführt. Als Ergebnis werden alle relevanten Informationen $\mathrm{zu}$ den gefundenen Optimalstellen ausgegeben. Zusätzlich wird der gesamte gefundene Lösungsraum grafisch in einem Diagramm ausgegeben (Abbildung 9). Dieses zeigt für jede gültige Lösung Regallänge und Regalhöhe und die dabei günstigste Gassenanzahl mit dem am besten geeigneten RBG. Die Lösungen sind je nach Investitionsaufwand unterschiedlich eingefärbt. Ähnlich gute Lösungen können somit vom Planer einfacher gefunden und betrachtet werden.

Wesentlich schneller als die vollfaktorielle Suche ist eine teilfaktorielle Suche, bei welcher sich eine spezielle Funktionseigenschaft der Zielfunktion zunutze gemacht wird. Diese besitzt in den Bereichen, in welchen die geforderte Stellplatzanzahl genau erreicht oder nur wenig überschritten wird, lokale Optimalstellen. Werden diese Stellen gezielt durchsucht, wird das globale Optimum zuverlässig identifiziert und die Anzahl der notwendigen Funktionsauswertungen deutlich reduziert. Diese Optimierungsmethode wird bei der Durchführung von Sensitivitäsanalysen eingesetzt. Bei diesen Parameterstudien werden die Auswirkungen von veränderten Planungsdaten auf ein optimal dimensioniertes Lager untersucht. Es ist möglich, die Auswirkungen unterschiedlicher Größenbeschränkungen, steigender oder fallende Leistungsanforderungen bzw. andere Stellplatzanforderungen zu untersuchen und zu visualisieren. Dadurch können die Robustheit der gefundenen Lösung bewertet und eventuell sinnvolle Lösungen mit leicht veränderten Rahmenbedingungen gefunden werden.

\section{$6 \quad$ Zusammenfassung}

Im Rahmen des Forschungsprojekts „Integrierte Lagersystemplanung“ wird eine neue Planungsvorgehensweise entwickelt, die den Planer in der Ausarbeitung und dem Vergleich von Planungsvarianten für automatische Lagersysteme unterstützt. Die Methode ermöglicht es, die Einflüsse unterschiedlicher Lagerkonfigurationen und Lagerstrategien auf die Umschlagsleistung zu berechnen und parallel dazu den Investitionsaufwand zur Realisierung der Planungsvariante zu schätzen. In einem rechnergestützten Werkzeug erfolgt die Eingabe der Daten und die Berechnung der Kenngrößen. Im Hintergrund wird dazu auf eine Datenbank mit Kosten- und Leistungsdaten der Lagerkomponenten zugegriffen. Durch die automatische Berechnung kann der Suchraum nach dem wirtschaftlich optimalen Lagersystem wesentlich erweitert werden. Über eine Optimierungsfunktion werden die Planungsvarianten optimal dimensioniert. Unwirtschaftliche Lösungen können somit 
schnell erkannt und sinnvolle Planungsvarianten näher betrachtet werden. Der Planer profitiert durch die Möglichkeit des einfachen und schnellen Vergleichs unterschiedlichster technischer Lösungen für einen spezifischen Anwendungsfall.

\section{Literatur}

[Alt02] Alt, W.: Nichtlineare Optimierung; Vieweg, Braunschweig, 2002

[Boz84] Bozer, Y. A.; White, J. A.: Travel-Time Models for Automated Storage/Retrieval Systems; In: IIE Transactions, 16 (1984) 4, S. 329-338

[Cha95] Chang, D.-T.; Wen, U.-P.; Lin, J. T.: The impact of acceleration/deceleration on travel-time models for automated storage/retrieval systems; In: IIE Transactions, 27 (1995) 1, S. 108-111

[FEM95] Regel 9.851 - Leistungsnachweis für Regalbediengeräte, Fédération européenne de la manutention, 1995

[Frö80] Fröhlich, G.: Optimierung der Lagerhöhe eines Hochregallagers; In: $\mathrm{F}+\mathrm{H}, 30$ (1980) 8, S. 670-671

[Gud73a] Gudehus, T.: Die Investition pro Palettenplatz; In: dhf (1973) 8, S. 506-509

[Gud73b] Gudehus, T.: Die optimale Höhe von Hochregallagern; In: dhf (1973) 2, S. $68-72$

[Gud72] Gudehus, T.: Grundlagen der Spielzeitberechnung für automatische Hochregallager; In: Deutsche Hebe- und Fördertechnik (1972), (Sonderheft), Nr. 18, S. 63-68

[Hur04] Hur, S.; Lee, Y. H.; Lim, S. Y.; Lee, M. H.: A performance estimation model for AS/RS by M/G/1 queuing system; In: Computers and Industrial Engineering, 46 (2004) 2, S. 233-241

[Hwa90] Hwang, H.; Lee, S. B.: Travel-time models considering the operating characteristics of the storage and retrieval machine; In: International Journal of Production Research, 28 (1990) 10, S. 1779-1789

[Joh96] Johnson, M. E.; Brandeau, M. L.: Stochastic Modeling for Automated Material Handling System Design and Control; In: Transportation Science, 30 (1996) 4, S. $330-350$

[Kra11] Kraul, R.: Ersatzmodelle für die Leistungsbewertung von automatischen Lagersystemen, Dissertation, Lehrstuhl fml, TU München, München, 2011

[Lee97] Lee, H. F.: Performance analysis for automated storage and retrieval systems; In: IIE Transactions, 29 (1997) 1, S. 15-28

[Lip03] Lippolt, C. R.: Spielzeiten in Hochregallagern mit doppeltiefer Lagerung, Dissertation, Fakultät für Maschinenbau, Universität Karlsruhe, Karlsruhe, 2003

[NN09] N.N.: Hochregallager Anwenderstatistik; In: Fördern und Heben, 59 (2009), Lager Marktbild 2009, S. 31-35

[Roo09] Roodbergen, K. J.; Vis, I. F. A.: A survey of literature on automated storage and retrieval systems; In: European Journal of Operational Research, 194 (2009) 2, S. 343-362

[Sar95] Sarker, B. R.; Babu, P. S.: Travel time models in automated storage/retrieval systems: A critical review; In: International Journal of Production Economics, 40 (1995), 2-3, S. 173-184 
[See06] Seemüller, S.: Durchsatzberechnung automatischer Kleinteilelager im Umfeld des elektronischen Handels; Dissertation, Utz, München, 2006

[VDI73] Regel 3561 - Testspiele zum Leistungsvergleich und zur Abnahme von Regalförderzeugen, Verein Deutscher Ingenieure, 1973

[VDI98] Regel 4480 - Durchsatz von automatischen Lagern mit gassengebundenen Regalbediensystemen, Verein Deutscher Ingenieure, 1998

Dieser Beitrag entstand im Rahmen des IGF-Vorhabens $15990 \mathrm{~N}$ „Integrierte Lagersystemplanung“ der Forschungsvereinigung Bundesvereinigung Logistik e.V. BVL, Schlachte 31, 28195 Bremen, das über die AiF im Rahmen des Programms zur Förderung der industriellen Gemeinschaftsforschung und -entwicklung (IGF) vom Bundesministerium für Wirtschaft und Technologie aufgrund eines Beschlusses des Deutschen Bundestages gefördert wird. 